\title{
Review on Electronic Correlations and the Metal-Insulator Transition in $\mathrm{SrRuO}_{3}$
}

Subeen Pang

Department of Materials Science and Engineering, College of Engineering, Seoul National University, Seoul 08826, Korea

*Correspondence to:

Pang $S$,

Tel: $+82-2-880-9316$

Fax: +82-2-885-9671

E-mail: subinf@snu.ac.kr

Received August 10, 2017

Revised September 12, 2017

Accepted September 18, 2017
The classical electron band theory is a powerful tool to describe the electronic structures of solids. However, the band theory and corresponding density functional theory become inappropriate if a system comprises localized electrons in a scenario wherein strong electron correlations cannot be neglected. $\mathrm{SrRuO}_{3}$ is one such system, and the partially localized $d$-band electrons exhibit some interesting behaviors such as enhanced effective mass, spectral incoherency, and oppression of ferromagnetism and itinerancy. In particular, a Metal-Insulator transition occurs when the thickness of $\mathrm{SrRuO}_{3}$ approaches approximately four unit cells. In the computational studies, irrespective of the inclusion of on-site Hubbard repulsion and Hund's coupling parameters, correctly depicting the correlation effects is difficult. Because the oxygen atoms and the symmetry of octahedra are known to play important roles in the system, scrutinizing both the electronic band structure and the lattice system of $\mathrm{SrRuO}_{3}$ is required to find the origin of the correlated behaviors. Transmission electron microscopy is a promising solution to this problem because of its integrated functionalities, which include atomic-resolution imaging and electron energy loss spectroscopy.

Key Words: $\mathrm{SrRuO}_{3}, 4 d$ orbital, Electronic correlation, Metal-Insulator transition, Transmission electron microscopy

\section{INTRODUCTION}

Classical electron band theory deals with noninteracting or weakly interacting electrons to introduce the concept of band structure (Imada et al., 1998). Electronic band structures form as a consequence of the periodic nature of solid lattices. According to the theory, metals can be easily distinguished from insulators. The highest bands of metallic substances, are partially filled or empty whereas those of insulators are completely filled by electrons with a substantial bandgap. If the bandgap is sufficiently small for the electrons in the highest band to be excited toward it at a finite temperature (i.e., indicating thermal excitation), the material is further specified as a semiconductor.

In this respect, electron band theory has successfully predicted the electronic properties of numerous solids. However, because the theory was established within weakly perturbative
Hamiltonians, it cannot reflect strong electron-electron correlations. The theory is not suitable for describing the physical natures of so-called strongly correlated materials. For instance, some transition-metal oxides with partially filled highest bands are not metallic, but rather exhibit insulating behavior (de Boer \& Verwey, 1937).

The importance of electron correlations in materials has thus emerged. What type of electron correlations can make a metallic compound insulating? One of the feasible explanations is Coulomb repulsion between electrons. If the noninteracting or weakly interacting Hamiltonian is perturbed by the repulsion, a metallic substance can become an insulator; this process is referred to as the Metal-Insulator transition (MIT).

Mott (1990), from the concept of Coulomb repulsion, explained how this kind of electronic correlation can induce an insulating phase. Imagine a perovskite-structured oxide

(a) This is an open-access article distributed under the terms of the Creative Commons Attribution Non-Commercial License (http://creativecommons.org/licenses/by-nc/4.0) which permits unrestricted noncommercial use, distribution, and reproduction in any medium, provided the original work is properly cited.

Copyrights @ 2017 by Korean Society of Microscopy 
with a transition metal at the B-site and the material has three electrons in $d$-originated $t_{2 g}$ orbitals. In the absence of electron-electron interactions, half-filled $t_{2 g}$ bands of the transition metals clearly indicate conducting behavior. However, when repulsion arises, electrons hopping from one transition-metal $d$ orbital to another would experience a strong Coulomb repulsion, because each $t_{2 g}$ orbital is already filled with a nonzero electron. If the repulsion is too large to be overcome, the electrons in the $t_{2 g}$ bands cannot move even if bands are only partially occupied. Eventually, the $d$ band is viewed as being split into two bands (Fig. 1): a band of three electrons occupying three $t_{2 g}$ states, and an empty band segregated by the repulsion strength. An insulating phase resulting from this type of the electron correlation is called a Mott insulator.

In a simple scheme, a MIT occurs when the total bandwidth of an original band is narrowed (resulting in a bandgap between split bands), when a certain number of electrons occupy a band, or when the effective strength of repulsion becomes substantial. These scenarios are often referred to as bandwidth-controlled, filling-controlled, and repulsioncontrolled MIT, respectively.

Apart from the Mott insulators, metallic materials with sizable correlations or at the proximity of the Mott insulating phases often have singular properties. Referred to as anomalous metallic phases, they have been found to exhibit diverse physical properties, such as strong fluctuations of spin states, high specific heat, and superconductivity. $\mathrm{SrRuO}_{3}$ (SRO) has been reported to be a correlated material in which the influence of electron correlations cannot be disregarded, despite the fact that actually it does not appear to be near to a Mott insulating phase in its bulk state, meaning that it

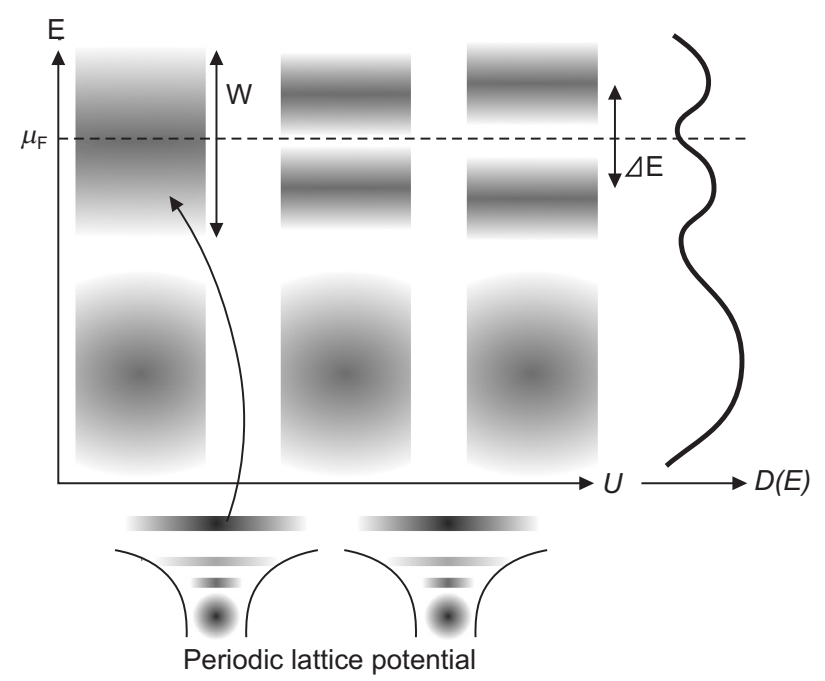

Fig. 1. Band structure perturbed by $U$ (Coulombic repulsion) and the corresponding density of states, $D(E)$. As $U$ increases, the system becomes an insulator. is only weakly correlated. Because $\mathrm{Ru}$ has a $d$ orbital that is substantially more dispersive than the those of conventional $3 d$ transition metals, $\mathrm{Ru}$-derived oxides will not exhibit extensive correlated behavior, compared to, for example, Mnor $\mathrm{V}$-derived oxides.

However, in certain environments, correlated behavior can be induced in SRO; for instance, SRO is known to exhibit nonFermi-liquid behavior, such as strong mass enhancement. In an ultrathin limit, the physical properties of SRO become peculiar. At a size approaching approximately four unit cells, SRO undergoes an MIT and it loses its itinerant, ferromagnetic property. Because the correlated behavior of SRO can be reasonably explained in terms of electronic repulsion, several treatises on SRO in Mott's language have been published. However, no prevailing and universal theory concerning the origin of abnormalities in SRO has been developed. Describing strong correlations in condensed matters is a very challenging subject.

\section{ELECTRONIC PROPERTIES OF BULK SRO}

\section{Oxygen Octahedra and Orthorhombic Structure}

At room temperature, SRO has an orthorhombic structure (Choi et al., 2010) with the pseudo-cubic lattice parameter $\alpha=0.393 \mathrm{~nm}$ (Kacedon et al., 1997). Because of the size difference between $\mathrm{Sr}^{2+}$ and $\mathrm{Ru}^{4+}$ (a kind of internal, inborn strain) and Jahn-Teller theorem (Jeng et al., 2006), the bulk state of SRO has an orthorhombic structure $(\mathrm{Pbnm})$ and $\mathrm{a}^{-}$ $\mathrm{a}^{-} \mathrm{c}^{+}$rotation (Kennedy \& Hunter, 1998; Woodward, 1997) of octahedra in Glazer notation (Fig. 2).

Consider an imaginary cubic SRO. Schematics of molecular orbitals in the cubic SRO are shown in Fig. 3. The Ru ion has valency of +4 and exists as $\mathrm{Ru}^{4+}$ in a fully ionic manner. If SRO has the maximum symmetry (i.e., a cubic structure), then the $t_{2 g}$ band would be a partially filled (four electrons)

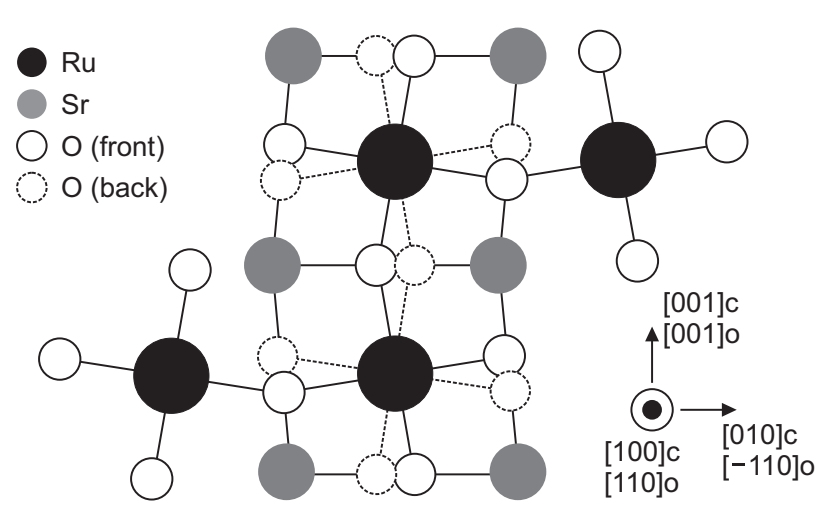

Fig. 2. Orthorhombic distortion in $\mathrm{SrRuO}_{3}$ (SRO) (Pbnm). Parameters $c$ and $o$ refer to the pseudo-cubic and orthorhombic unit cells respectively. Front and back indicate the positions of $\mathrm{Ru}$ atoms at which the corresponding oxygen atoms are bound. 
conduction band composed of three degenerate bands, as shown in Fig. 3, originating from $4 d$ orbitals of $\mathrm{Ru}$. The nearest conduction band is twofold degenerate. In addition, the $e_{g}$ bands have higher energy than the $t_{2 g}$ bands, and the energy of gap between the $t_{2 g}$ and $e_{g}$ bands in SRO is estimated to be approximately $3 \sim 4 \mathrm{eV}$.

When the rotational degree of freedom of the oxygen octahedra is relaxed, cubic SRO approaches its true ground structure. Suppose that rotation along only the pseudocubic [001] direction is allowed. In this case, SRO would become tetragonal, as shown in Fig. 3, and the degeneracy among Ru $4 d$ orbitals would be further destroyed accordingly. However, the orthorhombic or tetragonal perturbation is not sufficiently strong to form hard gaps between the bands in different symmetries and to overcome the crystal-field splitting of $t_{2 g}$ and $e_{g}$, irrespective of the occurrence of JahnTeller distortion. Moreover, the density functional theory (DFT)-based calculations have shown that the projected densities of the $t_{2 g}$-based states share similar energy levels (Dang et al., 2015; Ryee et al., 2016). Thus in SRO, the nearFermi bands are often tagged by $t_{2 g}$ and $e_{g}$ similar to the nearFermi bands of other cubic transition-metal oxides (e.g., $\mathrm{SrTiO}_{3}[\mathrm{STO}]$ ).

Nevertheless, because the orthorhombic distortion splits all five $4 d$ orbitals, each Ru ion in ordinary SRO prefers a certain electron orbit to the others. This tendency is referred to as

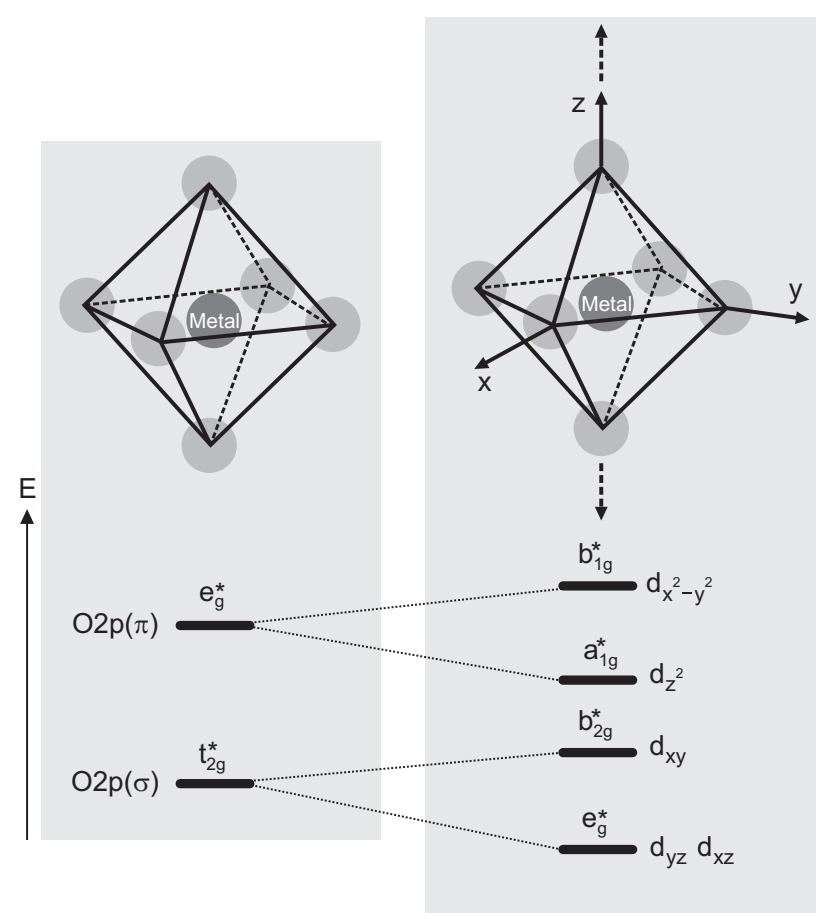

Fig. 3. Tetragonal descent in symmetry of oxygen octahedra and splitting of transition-metal $d$-orbitals. Unlabeled gray balls indicate oxygen atoms. Black dotted arrows indicate a tension along pseudo-cubic $c$ axis. orbital polarization or orbital ordering. However, as previously implied, the order of orbital ordering is not sufficiently large to induce a band gap in the $t_{2 g}$ orbitals (Jeng et al., 2006; Lin et al., 2008; Wang et al., 2009). The same is even true for $\mathrm{CaRuO}_{3}(\mathrm{CRO})$, in which the compound remains metallic regardless of a pronounced octahedral tilt. Exceptionally, in the case of $\mathrm{PbRuO}_{3}$, a strong hybridization between $\mathrm{Pb}^{2+} s p$ and $\mathrm{Ru}^{4+} t_{2 g}$ bands exists, which leads to the stabilization of weak but global orbital ordering of $\mathrm{PbRuO}_{3}$, opening a small band gap of $0.1 \mathrm{eV}$ at low temperatures (Kimber et al., 2009). In this respect, structural deformations such as octahedral tilt, internal misfit strain and orbital ordering barely become possible triggers of MIT (or formation of a band gap) in nonultrathin SRO unless additional dopant Ti atoms (Kim et al., 2005b; Lin et al., 2008) or a strong surface reconstruction (Jeng et al., 2006) is extant.

\section{Hubbard model}

One of the pioneering models to describe the systems of correlated electrons is the (single-band) Hubbard model, which can be considered as a simplified lattice fermion model:

$$
H=-t \sum_{i, j . \sigma}\left(\mathrm{d}_{\mathrm{i}, \sigma}^{\dagger} \mathrm{d}_{\mathrm{j}, \sigma}+\mathrm{d}_{\mathrm{j}, \sigma}^{\dagger} \mathrm{d}_{\mathrm{i}, \sigma}\right)+U \sum_{i=1}^{N}\left(n_{i \uparrow} n_{i \downarrow}\right)+\mu \sum_{i=1}^{N}\left(n_{i \uparrow}+n_{i \downarrow}\right)
$$

where $d_{i \sigma \sigma}^{\dagger}\left(d_{i, \sigma}\right)$ is the creation (annihilation) operator of an electron at lattice site $i$ and spin $\sigma, n$ denotes the corresponding number operator, and $t, U$, and $\mu$ express hopping from one site to another, on-site Coulombic repulsion produced by multiple electrons located at the same site, and the chemical potential of the given system, respectively. Parameter $U$ is also known as the Hubbard parameter.

Within the Hubbard model, so-called good metals and Mott insulators can be simulated. When $t>>U$, electrons in the lattice act totally freely and the corresponding band is only partially filled because of the Pauli exclusion principle. By contrast, if $t<<U$, the electrons at each site freeze to make the wavefunctions of the electrons hardly overlap each other because the total energy of the system can be decreased effectively when the effect of $U$ is minimized. Parameter $U$ is now tremendously larger than the width $(\propto t)$ of the original free-electron conduction band. When the parameters are changed from $t>>U$ to $t<<U$, the bandstructure will be shifted as described in Fig. 1 under the half-filled singleorbital model. The two split bands refer to the lower and upper Hubbard bands, respectively.

SRO is a poor metal, where $t \approx U$. Because $U$ is not large compared to the band width, the lower and upper Hubbard band overlap and Fermi-liquid behavior of electrons near the Fermi level (both bands contain the Fermi level, $E_{F}$ ) is conserved (Fig. 4A). However, this description is over- 
simplified. SRO has four electrons in the three $t_{2 g}$ variant orbitals. Thus, the electrons in one $\mathrm{Ru}$ site are also repulsive to each other (intrasite correlation, see Kanamori Hamiltonian in Georges et al., 2013). Suppose we try to insert four electrons into a $\mathrm{Ru}^{8+}$ site one by one. Until three electrons are inserted, no strong electronic repulsion would occur because the three electrons have different orbits $(x y, y z, z x)$ and are stabilized by maximized spin and angular momenta. However, when we add the fourth electron, this minority-spin electron should produce an energetically undesirable situation, e.g., intra orbital repulsion without spin-spin stabilization. Every possible state for the fourth electron is already filled with other electrons so that it immediately experiences a strong repulsion; therefore, the intra site density of states near the Fermi level becomes as described in Fig. 4B.

By generalization of (single-band) Hubbard model, $U$ develops into a parameter that is strongly related to both the intersite and the intrasite electronic correlations (Fig. 4C). As the effective strength of $U$ increases, the delocalization of the conduction band and the overlap between the major and minor $t_{2 g}$ bands interfere. If the magnitude of $U$ is insufficient to induce the Mott insulating phase of SRO, the minority-spin $t_{2 g}$ band (one-third filled) is the lowest conduction band. Consequently, SRO is referred to as a halfmetal (only the minority-spin band is conducting). However, the half-metallicity of SRO has not yet been demonstrated experimentally. In a photoemission spectroscopy (PES) experiment (Cox et al., 1983), the number of conducting electrons according to the density of states in SRO is shown to be 1 by assuming a free-electron gas condition for the electrons at the Fermi level, which is indirect evidence of halfmetallicity. However, in a recent study, Bern et al. (2013) failed to find an isotropic (minority) spin gas in an SRO thin film.

\section{Spectroscopic Evidences of Electronic Correlation}

The aforementioned results imply that the Hubbard repulsion energy is not as large as the crystal-field splitting, which reaches $3 \sim 4 \mathrm{eV}$, but nonetheless strongly influences the electrical properties of SRO. In this situation, the shapes of the PES and absorption spectra are expected to show

1) A lower Hubbard $t_{2 g}$ peak below the Fermi level.

2) A nonzero Fermi edge that originates from a single minor-spin electron.

3) An upper Hubbard $t_{2 g}$ peak above the Fermi level.

In actual experiments, the aforementioned features 1 and 2 are easily observed (Fujioka et al., 1997; Okamoto et al., 1999; Toyota et al., 2005). Furthermore, a large overlap exists between the oxygen $2 p$ and $t_{2 g}$ signals, justifying the importance of the role of oxygen atoms in SRO (Fujioka et al., 1997; Park et al., 2004). The unusual suppression of $t_{2 g^{-}}$ oriented signals compared with those predicted by DFT computations is interesting (Fujioka et al., 1997; Okamoto et al., 1999; Rondinelli et al., 2008). For example, in Fig. 5, the experimental spectra show both coherent and incoherent peaks (where the term incoherent refers to the antithesis of the coherent peaks of metals), whereas the coherent part is severely exaggerated in the DFT simulation results. Theoretically reproducing a correct-height $t_{2 g}$ peak is an interesting pursuit, and this phenomenon is often described as an effect of electronic correlation that we have not yet elucidated. In the terminology associated with dynamical mean field theory (DMFT), the appearance of a lower Hubbard signal becomes obvious because of the transfer of spectral weight from a quasiparticle peak of a weakly $U$-perturbated system to an incoherent peak of lower Hubbard band (Kotliar \& Vollhardt, 2004; Rozenberg et al., 1996).

In terms of the interband transition, the lower Hubbard band

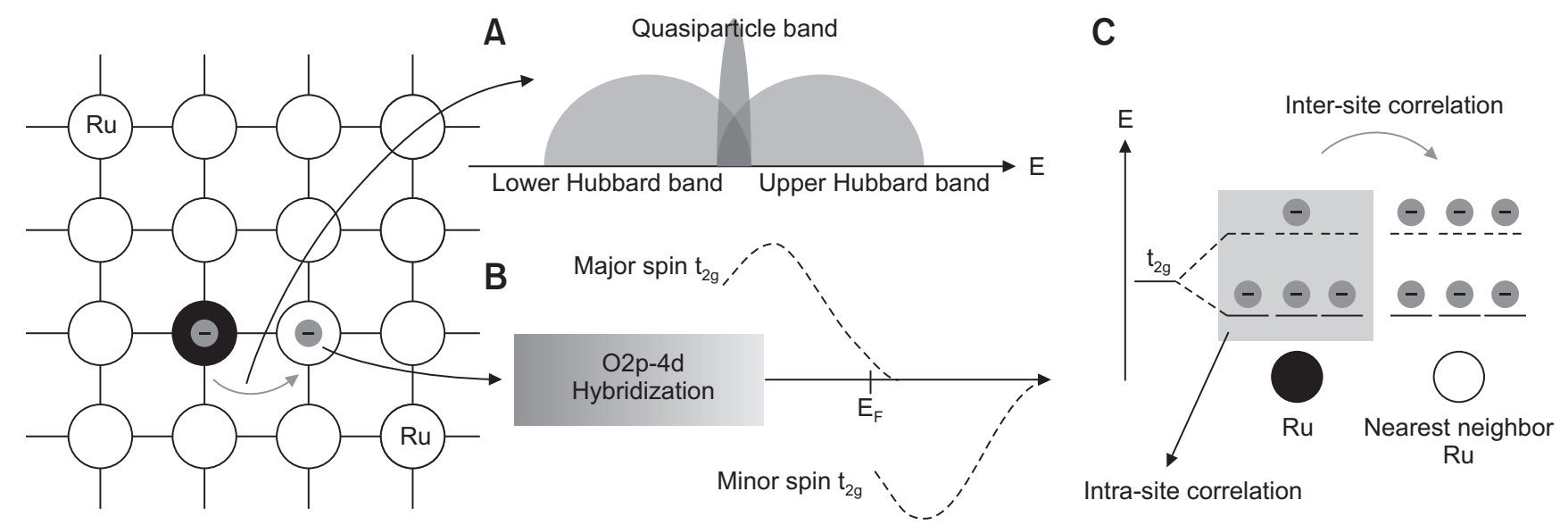

Fig. 4. Splitting of (A) a conduction band under the inter-site Hubbard repulsion and (B) the $t_{2 g}$ band in SRO because of the intrasite electronic repulsion. (C) As a result, one of the four $t_{2 g}$ electrons in SRO is located at the minor-spin $t_{2 g}$ band (black dotted states) and $t$ and $U$ control the delocalization of this electron. The orthogonal splitting among three $t_{2 g}$ bands is not sufficiently severe to break the degeneracy among them. 


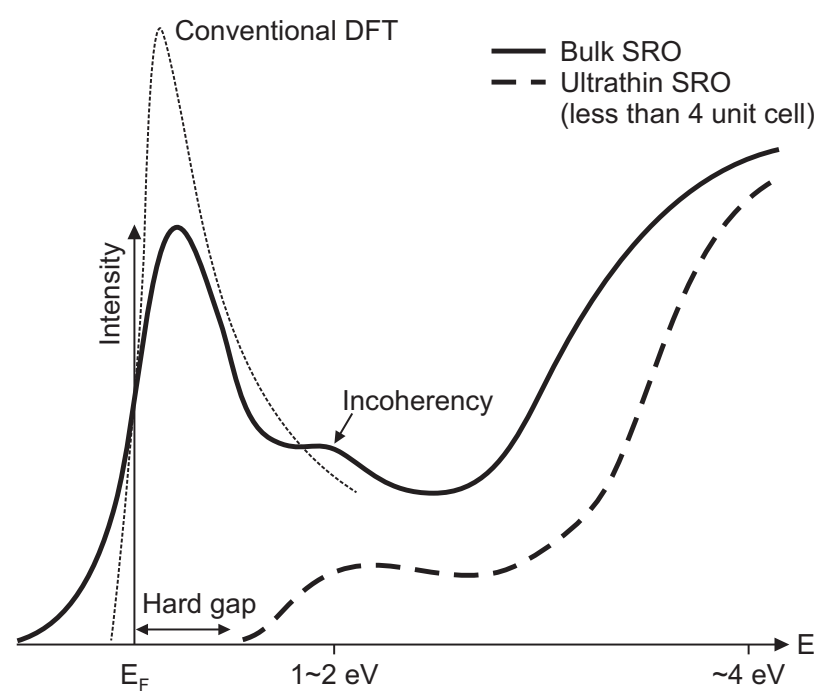

Fig. 5. Typical photoemission spectra of $\mathrm{SrRuO}_{3}(\mathrm{SRO})$. Incoherency and Fermi edge intensities are exaggerated for ease of comparison. Conventional density functional theory (DFT) calculations do not reflect the non-Fermi nature of the system and often overestimate the Fermi edge intensity, which is the very symbol of a metallic phase (Fujioka et al., 1997; Toyota et al., 2005).

(minority-spin $t_{2 g}$ band) is observed at approximately $1 \sim 2 \mathrm{eV}$ (Lee et al., 2001). Under the assumption that any meaningful signal in an optical conductivity signal of SRO is related to only the interband transitions, peaks observed within $10 \mathrm{eV}$ will consist of a very weak lower- $t_{2 g}$-to-quasiparticle/upper$t_{2 g}$ peak, a strong $\mathrm{O} 2 p$-to-upper- $t_{2 g}$ peak (because the lower $t_{2 g}$ band is full of electrons), and a strong O $2 p$-to- $e_{g}$ peak, among others (the terms weak and strong are based on Laporte's rule). Using this approach, we can again justify the existence of the lower Hubbard band in bulk SRO, located at approximately $1 \sim 2 \mathrm{eV}$.

The single-band Hubbard Hamiltonian can be exactly diagonalized if the well-known toy model is assumed for two half-filled sites. In this case, the bandgap between the lower and upper Hubbard bands is approximately $U$. Because the order of incoherency is approximately $1.2 \mathrm{eV}$ (Fujioka et al., 1997; Okamoto et al., 1999; Rondinelli et al., 2008), $U$ in SRO would be approximately $2.4 \mathrm{eV}$ (the appropriate value of $U$ is disputed, with values ranging from $1 \mathrm{eV}$ to 3 eV, e.g., as in Fujioka et al., 1997 and Kim \& Min, 2014). This value is lower than the bandwidth $(W)$ of the $t_{2 g}$ bands and the $t_{2 g}-e_{g}$ splitting. To ensure that the system is completely insulating, two spin-projected $t_{2 g}$ bands may not overlap each other, e.g., $U \approx 2 W$. Thus, although $U \approx 2 W$ is an oversimplified description, ordinary SRO is far from the Mott insulating phase and is referred to as a weakly correlated material. However, real-world materials have uncountable electronic correlations other than $U$. For instance, in principle, every material bears spin-orbit coupling, Hund's rule coupling, and electron-phonon coupling, although spin-orbit coupling in SRO is known to exert only a weak effect (Dang et al., 2015; Fujioka et al., 1997; Jeng et al., 2006). Hund's coupling, in particular, is considered an important correlation in SRO. Hence, SRO exhibits anomalous behaviors that cannot be explained by weakly correlated $U$ (Ahn et al., 1999). Some indicators that can be used to evaluate the degree of correlation are the effective mass and the (optical) conductivity because they reflect the electronic behavior of the conduction band. The effective mass of an electron trapped by high Hubbard repulsion approaches infinity because the electron cannot change its position from one $\mathrm{Ru}$ site to another (this phenomenon is said to be realized by the renormalization of the effective mass due to $U$ ). Quantitatively (Brinkman-Rice theory; Ahn et al., 1999),

$$
\frac{1}{m^{*}}=1-\frac{\left(U_{W}\right)^{2}}{\left(U_{W}\right)_{c}^{2}}, \quad U_{W}=\frac{U}{W}
$$

where $m^{\star}$ and $c$ are the effective mass of an electron and the critical value of $U_{W}$ at the border of the MIT. Under this theory, $U_{W}<2$ in SRO (Ahn et al., 1999) and, if $U \approx 2 \mathrm{eV}$, the mass enhancement does not exceed 5 (Dang et al., 2015). In addition, in a low $U$ limit, the conductivity-response function of the system will behave as (Dodge et al., 2000)

$$
\sigma(\omega)=\frac{A}{\left(\tau^{-1}-i \omega\right)^{\alpha}}
$$

within a low-frequency regime, where $\tau$ and $\omega$ denote the scattering time and the optical frequency, respectively. If $\alpha=1$, the above form of conductivity is Drudic because the real part of the response is proportional to $\omega^{-2}$. A typical character of the correlated materials thus shows a heavy effective mass and $\alpha \neq 1$ behavior (Dodge et al., 2000). The fact that SRO exhibits the optical response of an insulator (or even a local maximum in the response function at the proximity of 0 frequency) and a negative effective mass (Kostic et al., 1998) is peculiar. Therefore, the correlation effects in these systems do not depend on simple numerics. Structure, Hund's coupling, Coulombic interaction, and any other unrevealed interactions interplay, and this ensemble of external perturbations cannot be unveiled easily.

\section{Additional issues with spectroscopy}

Several authors have expressed pessimism about the very effect of the electronic correlations on the incoherency in SRO (Kumigashira et al., 2008; Siemons et al., 2007; Yang et al., 2016). In fact, factors related to defects in specimens cannot be ignored. Grain boundaries, nanostructure morphology, and oxygen vacancies can contaminate the systems undergoing spectroscopic analyses. The aforementioned authors mainly argue that 1) an inclusion of surface spectra and 2) offstoichiometry/morphological differences during specimen 
fabrication exaggerate the incoherency. The following is a compendium.

\section{Surface spectra}

The physics of surfaces at which the chemical discontinuity is abrupt is obviously distinct from that of the interface and bulk. A severe distortion of the band structure of SRO at the surfaces induces the transfer of spectral weight from Fermi edge quasiparticle peaks to the incoherent peaks (Liebsch, 2003a), which misleads that the specimen is expected to exhibit more sizable correlation effects than what it actually exhibits. The portion of the surface spectra cannot be fully removed from any spectroscopic data. Several authors have argued that previous studies dealing with uncapped specimens (bare surfaces) exaggerate the value of $U, 1.2 \mathrm{eV}$, and that it should be corrected to less than $1 \mathrm{eV}$ (Maiti et al., 2007b; Maiti \& Singh, 2005). In the case of the interfaces (where $\mathrm{Ti}^{4+}$ ions prevent abrupt discontinuity) and sufficiently thick specimens, the incoherency in the spectra is diminished (Kumigashira et al., 2008). However, Takizawa et al. (2005) have demonstrated a substantial spectral weight transfer even in a near-Fermi-level spectrum where the surface-driven effects are removed, and CRO still exhibited a non-negligible incoherent signal (Park et al., 2004; Yang et al., 2016), indicating that the Ru-based perovskites are possibly $U_{W^{-}}$-governed systems.

\section{Off-stoichiometry/morphology issues}

Similar to the enhanced incoherency at the surfaces, various experimental procedures that make SRO defective augment the incoherency. An artificial scrape (Kim et al., 2005a), $\mathrm{Ru}$-deficiency and poor deposition processes (Siemons et al., 2007) are the well-known cases, even changing the shape of core-loss interband transition spectra (Kim et al., 2004; Siemons et al., 2007). Other off-stoichiometry and morphological issues are involved in changes of the electric and magnetic properties of SRO; thus, they promote enhanced incoherency (Koster et al., 2012).

\section{Ferromagnetism in Bulk SRO}

Even if a Hamiltonian of a system does not contain spinoriented operators, Coulombic electron-electron correlation itself can reduce the spin degree of freedom of the system. For instance, in the Hartree-Fock approach, the ground state of a hydrogen molecule includes a triplet-spin component. Because the exchange integral (more generally, exchange correlation) between different (orthogonal) orbitals is positive. Hund's first rule, which states that a system with a maximum spin number is preferred, can be similarly deduced. Hubbard repulsion, or Coulomb repulsion, also induces a spin-related configuration, which is known as Hubbard ferromagnetism. A mean field version of ferromagnetism in the Hubbard model is well-known Stoner-type ferromagnetism. In the Stoner scheme, $U$ favors a magnetically ordered system because two electrons with opposite spins at the same site (band) produce additional repulsion energy. However, such magnetization induces a kinetic energy gain because of an excitation of majority-spin electrons. The kinetic energy gain is proportional to the density of states at the Fermi level. As a result, the Stoner criterion,

$$
U D\left(E_{F}\right)>1
$$

becomes a criterion for the spontaneous spin polarization, where $D\left(E_{F}\right)$ refers to the density of states at the Fermi level. Importantly, perturbing $U$ triggers the spin polarization. Suppose each $\mathrm{Ru}$ in SRO has one free electron and one conduction orbital. If $U$ is small $(t>>U)$, the system is stabilized mainly by the delocalization of $t_{2 g}$ electrons. Two electrons hopping into a same $\mathrm{Ru}$ site via wavefunction overlap (delocalization) should have opposite spin states because of the Pauli exclusion principle. Thus, in principle, a free-electron system with negligible electron correlations is paramagnetic. However, as $U$ becomes large, stabilization by delocalization and unfavorable Hubbard repulsion compete with each other because two overlapped electrons at the same $\mathrm{Ru}$ site give rise to an additional $U$ term. Imposing the same spin state on each electron keeps the electrons from being itinerant but can reduce the total Hubbard repulsion in the system. Thus, in a metallic state where $U$ and $t$ (or band width $W$ ) are comparable, $U$ promotes ferromagnetism.

SRO is an itinerant ferromagnet with a magnetic moment $1.0 \sim 1.6 \mu_{B}$ per formula unit (Kanbayasi, 1976; Mazin \& Singh, 1997; Rondinelli et al., 2008), which is smaller than net Bohr magnetron of an isolated unit cell, i.e., two-majority spin, $2 \mu_{B}$ (note that Grutter et al. [2010] have reported an exceptional value). This discrepancy is attributed to the itinerancy of the four $t_{2 g}$ electrons. The magnetic easy axis is the [001] of the pseudo-cubic lattice (Bern et al., 2013) and its Curie temperature is approximately $T_{C}=160 \mathrm{~K}$. Phenomenologically, the ferromagnetism of SRO is known to be well explained by Stoner theory when the external perturbations are negligible. However, as presented in the next sections, SRO even loses its itinerant, ferromagnetic property at the MIT border. According to the Stoner theory, no spontaneous magnetization or exchange splitting should occur at temperatures above $T_{C}$; nonetheless, a set of recent studies have reported the existence of a net magnetic moment $(M)$. However, whether the nonzero $M$ indicates a direct violation (Shai et al., 2013) of Stoner theory requires further investigation because the paramagnetic state can be regarded as a collection of randomly oriented local magnets induced by the spontaneous exchange splitting (Jeong et al., 2013). On the other hand, Han et al. (2016) and Kim \& Min (2015) also 
reported local and net magnetic moments at temperatures above $T_{C}$; however, the order of exchange splitting between $t_{2 g}$ orbitals rapidly becomes negligible with increasing $T$. Thus, the meaningful persistence of local exchange splitting beyond $T_{C}$ is doubtful. The degree of ferromagnetic order relies on not only the density of states (DOS) at the $E_{F}$ but also on the location of van Hove singularities and on the overall bandwidth (Han et al., 2016). Notably, oxygen-oriented local magnets are possible because of the $\mathrm{Ru} 4 d$ and $\mathrm{O} 2 p$ hybridization and because of the partial net spin moment in the oxygen atoms (Verissimo et al., 2012).

\section{ELECTRONIC PROPERTIES OF THIN-FILM SRO}

\section{Importance of Oxygen Atoms and Their Structure}

Despite the fact that $4 d$ electrons are dispersive, which makes the electronic correlations of SRO weak compared to other exceptional transition-metal perovskites, the $4 d$ orbitals of one $\mathrm{Ru}$ do not necessarily form a strong, direct band with those of another Ru. Both theoretically and experimentally, $4 d$-derived bands of $\mathrm{Ru}$ have been shown to include a considerable portion of oxygen $2 p$ orbitals (He et al., 2010; Verissimo et al., 2012). As such, the reasoning for using the word "hopping to" describe the behavior of the itinerant electrons of transitionmetal perovskites becomes clear. The four electrons in $t_{2 g}$ bands are not fully delocalized through the lattice but jump from $\mathrm{Ru}$ to oxygen and then again toward the next $\mathrm{Ru}$.

Therefore, not only the subtle nature of $4 d$ orbitals but also that of the oxygen atoms is responsible for the peripatetic physics of SRO because of the strong hybridization of $\mathrm{Ru} 4 d$ and $\mathrm{O} 2 p$ orbitals. Accordingly, the electronic structure of $t_{2 g}$-oriented bands is substantially coupled to the symmetry and structure of the lattice. For instance, if an artificial strain is imposed on SRO, the ordering of the $t_{2 g}$ bands and the magnetic state will vary, accompanied by a distortion of oxygen octahedra ( $\mathrm{Gu}$ et al., 2012). CRO provides another example of the importance of the $\mathrm{Ru}-\mathrm{O}-\mathrm{Ru}$ bonding. $\mathrm{CRO}$ is also an $\mathrm{ABO}_{3}$-type ruthenium oxide-based perovskite. Its $\mathrm{Ru}-$ $\mathrm{O}-\mathrm{Ru}$ channel is more distorted than that of SRO, because $\mathrm{Ca}^{2+}$ has a smaller ionic radius than $\mathrm{Sr}^{2+}$, which makes the $t_{2 g}$ electrons more localized and the $\mathrm{Ru}-\mathrm{O}-\mathrm{Ru}$ channel more distorted. These effects result in a narrow $t_{2 g}$ band, suppression of quasiparticle signals, and increased on-site Hubbard repulsion. The effect is obvious (Dang et al., 2015; Han et al., 2016; Takizawa et al., 2005; Yang et al., 2016). The signal of the incoherency is enhanced, whereas that of the quasiparticle is subdued, which may explain the MIT of SRO at the ultrathin limit. Ultrathin SRO is usually deposited onto an STO substrate, which induces compressive strain in the SRO. The effect of the strain can become a global physics of SRO when $\mathrm{SRO}$ approaches its ultrathin limit. If the structural issue is in fact influential, it can induce an MIT without changing $U$, as discussed in the next sections.

\section{Thin-Film Structure of SRO}

Thin-film SRO exists in a completely different environment than the bulk state. Several studies have emphasized the importance of the role of interfaces, e.g., SRO/STO. An STO substrate (usually [001]) exerts a compressive strain on the SRO thin film because the lattice constant of cubic STO is smaller than that of pseudo-cubic SRO, and this SRO/ STO model system shows some interesting phenomena. For example, an orthorhombic-to-pseudo-orthorhombic or orthorhombic-to-tetragonal transition of SRO emerges as the system reaches the ultrathin and low-oxygen-vacancy limit (Chang et al., 2011; Lu et al., 2013a, 2013b; Vailionis et al., 2008). Notably, two possibilities exist for the direction of the pseudo-orthorhombic or tetragonal $c$ axis: pseudocubic [100] or [001]. The key factor for such structural transitions is suppression of the rotation and tilt of the oxygen octahedra because the structural degree of freedom is restrained by the interfacial strain (Chang et al., 2011). For example, because of the strong in-plane strains, the only possible degree of freedom becomes the rotation along the pseudo-orthorhombic $c$ axis. Fig. 6B is an extreme case where the pseudo-orthorhombic $c$ axis is along the pseudocubic [001] and the tetragonal transition is fortified. This system refers to either $a^{0} a^{0} c^{-}$or $a^{0} a^{0} c^{+}$(Glazer notation). One easy approach to observe the (tetragonal) anisotropy is to evaluate the (directional) magnetization curves. If the pseudo-orthorhombic and cubic $c$ axes are parallel, the inplane spontaneous magnetizations are oppressed, whereas the magnetization along the pseudo-cubic [001], which is the magnetic easy axis of SRO, remains magnetically ordered (Lu et al., 2013a, 2013b). Given that the ferromagnetism of SRO relies on the Ru-O-Ru channel and that the $\mathrm{Ru} 4 d$ and $\mathrm{O}$ $2 p$ hybridization is important (He et al., 2010), the electrical and magnetic anomalies are reasonably speculated to arise from the interfacial strain and the structural phase transitions (Vailionis et al., 2008). If the orthorhombic distortion of bulky SRO is so large that the Ru-O-Ru bonding cannot be maintained, the system will have an extremely small hopping parameter $t$ and the ferromagnetism will disappear. However, as mentioned in the previous sections, the $4 d$ electrons in bulk SRO are neither heavily localized nor correlated. Thus, the aforementioned abnormalities represent the strong effect of the tetragonal transition.

However, some studies have cast doubt on the aforementioned contentions; that is,

1) The distortion of octahedra and suppression of the magnetic moment persist within only $1 \sim 3$ unit cells from the interface (He et al., 2010).

2) The ferromagnetic ordering remains even if SRO 
A

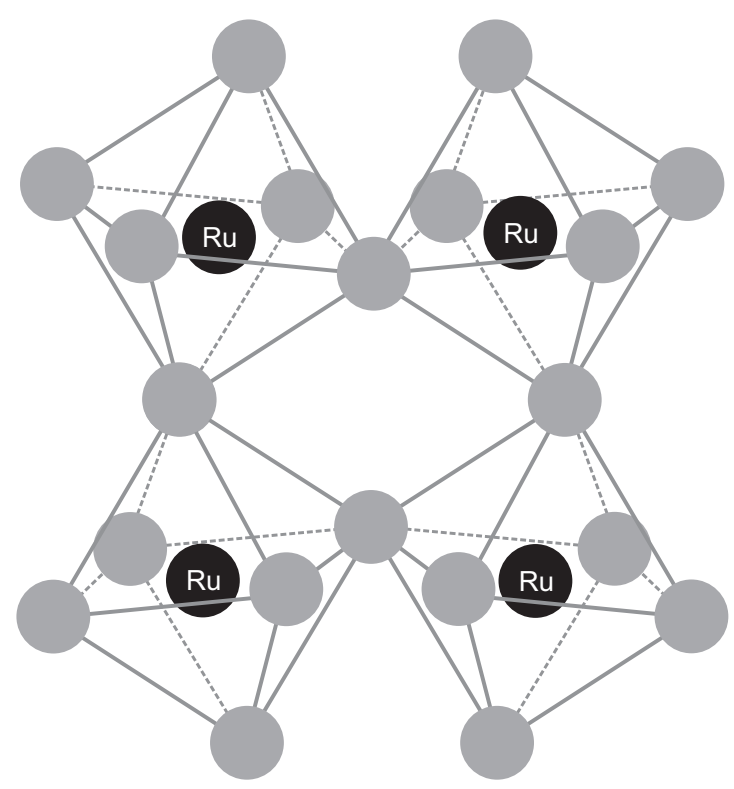

B

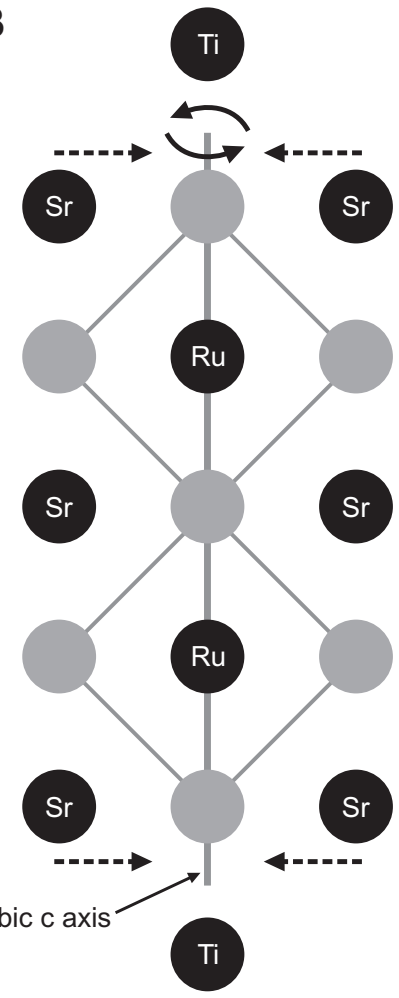

Fig. 6. (A) Orthorhombic lattice system caused by tilt, rotation, and Jahn-Teller distortion of oxygen (gray circles without labels) octahedra. (B) When compressional strains (black dotted arrows) from $\mathrm{SrTiO}_{3}$ (STO) interfaces exist, one of the energetically favored lattice distortions at the interfaces is the rotation of the octahedra along the pseudocubic c direction, as shown in (B) (black arrows).

reaches 1-unit-cell thickness, and the in-plane compressive strain increases the magnetic moment per $\mathrm{Ru}$ atom (Gu et al., 2012).

Nonetheless, the speculation that the tilt of the octahedra along the pseudo-orthorhombic $\mathrm{c}$ axis is suppressed near the interface is reasonable. The problems that make a simple description nearly impossible are the electrical and magnetic subtleties. In the single-band approximation, the bandwidth of the Hubbard bands $W$ is $\sim z t$, where $z$ is a coordinate number. If we assume SRO is under compressive in-plane strain, then, according to crystal-field theory, the energy of the $d_{x z, y z}$ orbitals will be lowered and the $\mathrm{Ru}-\mathrm{O}-\mathrm{Ru}$ channel will remain intact (bonding angle $\sim 180^{\circ}$ ). However, because of the elongation along the pseudo-cubic $c$ axis, $t$ in this direction decreases, resulting in a narrowed bandwidth and diminished $\mathrm{Ru}-\mathrm{O}$ overlap. Therefore, anticipating the behavior of each $t_{2 g}$ orbital under external strain is difficult (see, e.g., Fig. S7 in the supplementary material of Gu et al., 2012).

\section{Antiferromagnetism in SRO}

SRO has been reported to become antiferromagnetic at its ultrathin limit, although this behavior needs further study and review (Xia et al., 2009). In fact, magnetism in SRO is very subtle. In numerical studies, the ferromagnetism of SRO cannot be maintained if a magnetic long-range ordering term is excluded (Etz et al., 2012; Kim \& Min, 2014), and the Ru-O$\mathrm{Ru}$ channel is severely distorted. The resultant ground state is nonmagnetic or even antiferromagnetic. Thus, the effects of interfaces (Gu et al., 2012), reconstructions at surfaces (Gupta et al., 2014; Kim \& Min, 2014; Mahadevan et al., 2009), and the antiferromagnetism in the ultrathin systems may be related to each other. In fact, the localized $4 d$ electron system has some reasons to be antiferromagnetic.

As $U$ induces autonomous ferromagnetism under the $U \approx \mathrm{t}$ condition, it also engenders antiferromagnetism in a localized, $U>>t$ system. In this case, $\mathrm{t}$ acts as a perturbation instead. The second-order perturbation term indicates that the ground energy of the fully localized system is lowered by an order of $t^{2}$. However, if the electrons in this system are ferromagnetically spin-polarized, they cannot be overlapped irrespective of the value of $t$, because two electrons with the same spin state and at one localized site violate the Pauli exclusion principle. Hence, electron hopping can stabilize the localized eigenstate, which favors the antiferromagnetic configuration, which is known as Mott antiferromagnetism or direct exchange antiferromagnetism.

However, Mott antiferromagnetism is not fully germane to the antiferromagnetic properties of transition-metal perovskites because we cannot describe the intersite interaction without intermediate oxygen. Thus, a common mechanism for the antiferromagnetism of transition-metal perovskites is socalled superexchange; the exchange interaction between d-electrons of each site is provoked via hopping toward the nearest oxygen $p$ orbital. In fact, antiferromagnetism of 
SRO has been modeled on the basis of the superexchange interaction in theoretical studies (Etz et al., 2012; Mazin \& Singh, 1997). Though the detailed background of superexchange differs from that of Mott antiferromagnetism (e.g., the Goodenough-Kanamori rules), they share a similar concept in that two overlapping electrons cannot have the same spin state.

\section{MIT AT THE ULTRATHIN LIMIT}

Ferromagnetism and the DOS at the Fermi level of SRO have been predicted to be relevant (e.g., the Stoner model). In fact, a significant suppression of itinerant ferromagnetism, zero Curie temperature, and a loss of metallic behavior appear almost concurrently as the thickness of the SRO layer approaches approximately four unit cells. Several mechanisms of MIT exist in Ru-based perovskites, including Anderson localization due to highly disordered electrical potentials in the lattice (Kim et al., 2005b), a structural phase transition induced by varying the temperature (Gorelov et al., 2010; Kimber et al., 2009), and a charge-transfer MIT (Williams et al., 2006). The details of each mechanism differ but they do share common theoretical backgrounds; Mott-Hubbard and crystal-field theories. In this review, thickness-driven MIT is the main topic.

\section{Discrepancies among Experimental Results}

MIT in ultrathin SRO is represented by two states, ferromagnetic metal and nonmagnetic/antiferromagnetic insulator states. However, the exact thickness at which the MIT begins is controversial. Every ultrathin ( $<10$ unit cells) SRO is virtually located at the vicinity of the insulating phase, and MIT appears within only one unit-cell difference. Because the physics of such a tiny system is certainly susceptible to unwanted and unpredicted external agitations, the MIT induced by a "one unit-cell difference" is, in fact, very subtle. An example is presented by Gu et al. (2012). The SRO/STO (1/1) superlattice preserves a weak hysteresis loop though the STO layers, substantially impeding the interlayer overlap between the $4 d$ bands of $\mathrm{Ru}$. Unless otherwise indicated, the SRO discussed in this section is deposited on STO.

PES is a useful tool for evaluating MITs, because it can detect the band structure (and possibly the electronic correlations) directly. At a unit-cell thickness of four, the Fermi edge spectrum is completely shrunk, opening a weak band gap (Toyota et al., 2005) (Fig. 5). Incoherent spectral weight transfer also occurs, and the position of the incoherent peak remains approximately $1.2 \mathrm{eV}$. Corresponding resistivity data show that the resistivity of specimens with a thickness less than four unit cells is inversely proportional to $T$ and that its absolute value increases (Toyota et al., 2005, 2006). The resistivity data of Shen et al. (2015) somewhat coincide with those of Toyota et al. (2005, 2006); however, Shen et al. (2015) assumed that the MIT originated as an electron-phonon interaction. A more recent PES and X-ray magnetic circular dichroism (XMCD) study has indicated a similar behavior, where the border of the MIT amounts to a unit-cell thickness of 3-4 (Ishigami et al., 2015).

In Xia et al. (2009), the MIT border is between three and four monolayers. The strong suppression of the ferromagnetic hysteresis loop, zero Curie temperature (as also reported in Toyota et al., 2005, 2006), inversely proportional $T$ dependence, and increase of the absolute value of resistivity all indicate the emergence of the conspicuously insulating phase. Interestingly, Xia et al. (2009) addressed the possibility of an antiferromagnetic insulator detected by the reminiscence of the exchange-bias effect. Chang et al. (2009) observed similar insulating behaviors, such as a zero Curie temperature and an absolute increase of resistivity; however, the resistivity did not exhibit the inverse $T$ dependence, and MIT occurred at a thickness between two and three unit cells. This discrepancy may be derived from morphological differences. The atomic force microscopy (AFM) images of Chang et al. (2009) and Toyota et al. (2005) display an apparently distinct surface morphology, whereas those of Chang et al. (2009) and Xia et al. (2009) show some similarities. Chang et al. (2009) reported a further decrease in free-carrier density as the thickness reached four unit cells.

Bern et al. (2013) reported that thinner SRO layers result in weaker magnetic moments and hysteresis loops. The decrease of the Curie temperature begins at a thickness less than three unit cells; however, the complete disappearance of the local magnetic moment and ferromagnetism would be achieved only at a thickness of less than two unit cells. Strong increases in the resistivity and its inverse- $T$ dependence occur within two unit-cell-thick samples. Interestingly, the magnetic easy axis changes from the out-of-plane direction to the in-plane direction at a unit-cell thickness of two.

In terms of the superlattice (STO/SRO), both (3/3) (Bern et al., 2013) and (1/1) (Gu et al., 2012) superlattices show highly suppressed, but not zero, spontaneous magnetization. As already mentioned, because STO is a nonmagnetic insulator with a $3.2 \mathrm{eV}$ band gap and because the extent of the $\mathrm{Ru} t_{2 g}$ band is effectively limited by the oxygen atoms, the behaviors of SRO thin layers in superlattices should be similar to the behaviors of SRO thin films. Accordingly, the resistivity of the (3/3) STO/SRO superlattice is inversely proportional to $T$, meaning that it is insulating. Liu et al. (2012) have addressed $\left(\mathrm{LaAlO}_{3}=\mathrm{LAO} / \mathrm{SRO}\right)$. Unlike (STO/SRO), (LAO/SRO) with an intermediate thickness ( $3 \sim 10$ unit cells) is an insulator only at low temperatures. The system transforms into a totally insulating system as it approaches a (2/2) superlattice. Overall, SRO undergoes a MIT at thickness between two and four unit cells; the reported characteristics are 
1) An absolute increase in the resistivity,

2) An inverse $T$ dependence,

3) Shrinkage of spontaneous magnetization, and

4) Access to a zero Curie temperature,

where not all experiments are in perfect concordance.

\section{Theories of MIT}

\section{Difficulties in the DFT simulations}

Even if the bulk SRO physics can be explained using MottHubbard type correlations, the state of insulating SRO need not be a Mott-Hubbard insulator with a considerable $U$. In PES studies, whether the position of the incoherency shows a meaningful movement from the Ru-characteristic $1.2 \mathrm{eV}$ (Yang et al., 2016) to $2.0 \mathrm{eV}$ (Ishigami et al., 2015) or not (Toyota et al., 2005) is debatable. Given that $U$ should be much higher than the bulk value to make the system insulating, the PES results show that ultrathin SRO is not a pure Mott insulator. The localized state can be realized by factors other than a $U_{W}$. For example, a structural distortion may play a critical role, and the other possible mechanisms remain to be checked. Interestingly, the disappearance of ferromagnetic behavior exists at the boundary of the insulating state. According to the Stoner criterion, the presence of zero DOS at the Fermi level removes the energetic advantage of the ferromagnetic state.

However, even though the Stoner model and the corresponding ab initio calculations have been used to successfully model the bulk magnetic properties of SRO, simple DFTbased approaches fail to approximate the MIT and the incoherency from $U$ (e.g., Fig. 5). One of the studies dealing with the subtle behavior of the MIT of SRO on the basis of DFT is that of Rondinelli et al. (2008), who demonstrated the poor quality of DFT $+U+$ correction. The metallicity arises from the minority-spin $t_{2 g}$ band (as discussed, it has not been proven experimentally). The $t_{2 g}$ band with major spin electrons is obviously located beneath the $E_{F}$, and a hard gap exists between major spin bands. Whereas Rondinelli et al. (2008) found the antiferromagnetic configuration at a unit-cell thickness of two and described the bulk properties using $U_{\text {eff }}=1 \sim 2 \mathrm{eV}$ ( $U_{\text {eff }}=U-J$, where $J$ is Hund's coupling parameter), this approach is barely successful when applied to model the complete removal of a partially filled minor $t_{2 g}$ band near the Fermi energy. In principle, a high $U$ can reproduce the formation of a hard gap but it will result in an incorrect bulk phase (Dang et al., 2015). For example, the high $U$ condition indicates an insulating $\mathrm{V}$-oxide even if it is at the metallic side of the MIT (Liebsch et al., 2005). In addition, even if $3 \sim 4 \mathrm{eV} U$ (in certain situations) induces the disappearance of delocalized bands near $E_{F}$, it can incorrectly predict magnetic behaviors in SRO (Gu et al., 2016).
Broken symmetry at surfaces and interfaces

Because the specimens in several studies used were epitaxially deposited and exposed to vacuum (or air) directly, considering the effects of surface reconstruction, i.e., broken symmetry, is reasonable. Although the existence of antiferromagnetism, as proposed by Xia et al. (2009), is still controversial, surface reconstruction facilitates the emergence of both antiferromagnetic behavior and a hard gap near the $E_{F}$ (Autieri, 2016; Gupta et al., 2014; Jeng et al., 2006; Kim \& Min, 2014). The two main effects of surface reconstruction are described as follows:

1) Because of the difference in sizes of $\mathrm{Ru}$ and $\mathrm{Sr}$, oxygen atoms tend to deviate from their original positions (Kim \& Min, 2014). This deviation usually results in the destruction of O-Ru-O channel and the corresponding antiferromagnetism and narrow $t_{2 g}$ band. We reiterate that ferromagnetism in SRO is subtle and needs a wellestablished long-range force (Etz et al., 2012).

2) Surface reconstruction leads to a substantial change in the crystal field toward Ru. Alongside the decrease in symmetry of the Ru octahedra, anomalous $d$-orbital ordering occurs and a hard gap opens between the orbitals. However, the $d$-orbital ordering is not solely dependent on the crystal field and its mechanism has not been clarified. Worse yet, DFT $+U+$ surface reconstruction can overestimate the antiferromagnetic state (Mahadevan et al., 2009).

The authors of previous studies consider the surface reconstruction to be important because such a precipitous environment can induce an MIT even within the DFT $+U$ scheme. As already mentioned, DFT $+U$ does not guarantee an MIT under ordinary circumstances. The work of Verissimo et al. (2012) is one of the extremes, where SRO remains halfmetallic and ferromagnetic at one-unit-cell thickness (SRO/ STO supercell). Bern et al. (2013) refute this result because no two-dimensional electron gas exists inside the (1/1) supercell in the experiment. Within the interfaces, high (tensile) inplane strain is needed to induce antiferromagnetic insulator behavior (Gu et al., 2012). However, the MIT exists regardless of the presence of the surface construction (e.g., MIT in a superlattice (Bern et al., 2013; Liu et al., 2012) and in a layered structure [Tian et al., 2007]). One possible explanation for the MIT in capped (i.e., STO/SRO/STO) SRO samples is that $\mathrm{DFT}+U$ underestimates the interfacial relaxation and reflects $U$ inefficiently. The tetragonal transition may govern the physics of ultrathin SRO, i.e., at least two-third of three unitcell SRO would be deformed according to the discussions in the previous sections. In this case, the insulating phase may have originated from the interfaces.

\section{Dimensionality issues}

SRO itself is not a material governed by Mott physics and only 
weakly correlated (Dang et al., 2015; Georges et al., 2013; Yang et al., 2016). During the MIT, the degree of correlation should increase, or that of $W$ should decrease according to the MottHubbard picture. If $U$ remains small compared to the bulk $W$, then $W$ likely changes as a result of the MIT. In fact, the W of the $t_{2 g}$ band can decrease because of the broken periodicity of ultrathin specimens, which results in a Mott insulator. SRO has a van Hove singularity at the proximity of $E_{F}$ (Dang et al., 2015; Han et al., 2016), and as the thickness approaches its ultrathin limit, where the dimensionality of the system changes from $3 \mathrm{D}$ to $2 \mathrm{D}$ (or even $1 \mathrm{D}$, depending on the shapes of d-orbitals in $t_{2 g}$ symmetry), the DOS at the $E_{F}$ decreases (Chang et al., 2009; Verissimo et al., 2012). Similarly, $\mathrm{SrVO}_{3}$ (SVO) undergoes a MIT at its ultrathin limit, and the MIT is ascribed to the shrunken dimensionality and corresponding van Hove peaks near the $E_{F}$ (Gu et al., 2013; Liebsch, 2003b; Yoshimatsu et al., 2010). Such a low-dimensional system also has low $t_{2 g}$ orbital degeneracy, making $\left(U_{W}\right)_{c}$ smaller than the bulk value (Gunnarsson et al., 1996). However, no direct evidence of 2D or 1D quantum confinement effects (e.g., multiple small peaks near $E_{F}$ originating from integersplit band structures, as reported by Zhong et al. (2013) and Yoshimatsu et al. (2011), which is one of the typical features of low-dimensional systems. Furthermore, Zhong et al. (2015) have claimed that the mere non-periodicity of the interfaces and surfaces cannot produce the insulating SVO even in the DFT+DMFT scheme.

\section{Hund's coupling}

Dang et al. (2015) have shown that the correlated behavior of SRO originates from $J$ as well as $U$ and that the quasiparticle mass enhancement, lifetime, and self-energy are highly sensitive to J. SRO is, in this view, a Hund's metal. This description gives a fairly successful explanation for the tricky SRO physics without reaching the Mott physics region (Dang et al., 2015; Georges et al., 2013). However, J does not necessarily lead the system to an insulating state. Rather, as long as the delocalized band is not half-filled (e.g., three electrons in a $t_{2 g}$-oriented band), J increases $\left(U_{W}\right)_{c}$. The reason becomes obvious when one treats the atomic limit of orbitals, where

$$
\Delta_{a t}=E_{0}(N+1)+E_{0}(N-1)-2 E_{0}(N)=U-3 J
$$

is the energy required to transfer an electron from one atom to the other under Kanamori Hamiltonian if $E_{0}(M)$ is the energy of an atom with $M$ electrons (Georges et al., 2013). Even if an additional electron suffers from $U$, J compensates for it by spin-spin (same spins) interactions (imagine two $t_{2 g}$ sites, each with two electrons). As a result, thin SRO can be regarded as a bad metal or correlated metal, induced by $J$, but not at the vicinity of a Mott insulator.
$J$ and crystal-field splitting compete with each other. In simple terms, $J$ prefers energetically degenerate $t_{2 g}$ orbitals (this will result in the maximal spin moment) and the crystal-field splitting tends to decouple them. Again the energy required to transfer an electron is

$$
\Delta_{a t}=U-5 \mathrm{~J}+2 \Delta(2 \Delta>3 J, \text { atomic approximation })
$$

in the presence of crystal-field splitting term $\Delta$ and when $2 \Delta>3 J$ (Georges et al., 2013). $J$ is clearly observed to lower the energy barrier, driven by $\Delta$. In addition to this feature, because the energy gap between the largest spin-moment state (the ground state in terms of $J$ ) and its spin-multiplet variants widens as $J$ becomes large, $J$ hinders orbital-orbital fluctuations and thus hybridizations (de'Medici et al., 2009). As a result, the three $t_{2 g}$ orbitals become independent of each other. In this way, the so-called orbital selective Mott transition (OSMT) arises with the presence of $J$ (Ferrero et al., 2005; Georges et al., 2013). If the bandwidths of the orbitals differ (e.g., because of interfacial effects), OSMT is promoted (Koga et al., 2004), i.e., narrow orbitals become insulating first. Because of the Hubbard repulsion and enhanced localization of orbitals, the loss of symmetry induced by $\Delta$ produces a prominent effective splitting $\Delta_{\text {eff }}$ (Poteryaev et al., 2007, 2008). A DFT+DMFT study has shown the antiferromagnetic and insulating STO/SRO/STO with one-unit-cell thickness ( $\mathrm{Si}$ et al., 2015), with a decent $\Delta_{\text {eff }}$ between $d_{x y}$ and $d_{x z, y z}$.

\section{Final remarks}

On the basis of earlier discussions, the influences of $U, J$, $\Delta$ (descent in symmetry), and dimensionality should be considered to address the MIT in SRO. At a minimum, the MIT occurs in a capped SRO with a thickness of two to four unit cells. Although $U$ is not sufficiently large to induce the Mott insulating state, $\Delta$ and $J$ can hinder the intersite and intrasite orbital hybridizations, which produces a hard gap in the minor $t_{2 g}$ band. However, the effect of $\Delta$ on the anomalous orbital ordering is not obvious (i.e., the in-plane compressive strain may or may not simply increase the energy of the major $d_{x y}$ band), and different authors have proposed different views (e.g., refer to Gu et al., 2012; Gupta et al., 2014; Si et al., 2015). In addition, the authors of some reports argue that electronphonon coupling is an important factor. Both experiments (Shen et al., 2015) and computations (Yang et al., 2016) can provide clues concerning the correlating nature of electrons and phonons; however, the degree of coupling at high temperatures is under question.

\section{TRANSMISSION ELECTRON MICROSCOPY AND MIT IN SRO}

Among the possible origins of the MIT discussed to this 
point (i.e., structural deformations caused by vacuum or substrates (change in symmetry), dimensionality issues, and the cooperation of internal parameters, $U, J$, and $\Delta$ ), some mechanisms exist that can induce a MIT, including

1) Charge transfer of $\mathrm{Ru}$ and other atoms (Chen et al., 2013; Jang et al., 2011; Williams et al., 2006; Yoo et al., 2015);

2) Anderson localization (disordered potentials) (Kim et al., 2005b; Maiti et al., 2007a),

although their influences are expected to be small (e.g., no specific evidence of charge transfer between $\mathrm{Ru}$ and $\mathrm{Ti}$ [Kim et al., 2005b]); because PES experiments have not revealed any noticeable multiplet signals associated with different valences, we are open to all possibilities for the hidden physics of the MIT. It is an ensemble of such effects. The previous sections included descriptions of PES, X-ray absorption spectroscopy (XAS), AFM, and XMCD being used to find the very origin of MIT in SRO; however, none of them establishes the full picture correctly. As mentioned, all aspects of MITs cannot be explained using only one experimental technique. Furthermore, the phase boundary is located below four unit cells; thus, the specimens should be processed with meticulous care if one cannot guarantee fine spatial resolution.

The main source of MIT appears to be attributable to the $t_{2 g}$ band structure. Even though the effect of $J$ and the exchange correlations is hardly reflected on non-spin-resolved instruments, the photonic signals display the crude shapes of the valence and conduction bands at the low, core (Kim et al., 2004), and near Fermi-edge energy levels. Actually, the photonic experiments succeed in presenting the vestiges of $U$ and the formation of a hard gap. However, few researchers would agree with the idea that $U$ itself is the cardinal driving force of MIT. The localization effect of moderate $U$ is not large; thus, researchers investigate the influences of the dimensionality issues, charge transfer, and disordered potentials such as the surface reconstructions and unwanted vacancies. Again, these factors cause the lattice deformation and global strains. The experiments, e.g., PES, cannot clarify these concepts, suffering from ex situ contamination.

On the basis of the aforementioned discussions, transmission electron microscopy (TEM) (Fig. 7) becomes a key technique for studying the MIT of SRO. Suppose we want to observe the origin of the MIT in specimens $\mathrm{C} / \mathrm{SRO} / \mathrm{C}(\mathrm{C}=$ cap) to prevent abrupt surface reconstructions. The prime feature of TEM is its exquisite spatial resolution. Structural information about C, SRO, and even interfacial regions in the ultrathin films can be obtained, as shown in Fig. 7 and 8. In addition, by confirming the interfacial atomic arrangements, the impact of Anderson localization and $\Delta$ can be guessed. Using electron energy loss spectroscopy (EELS), integrated with TEM, we can portray, under certain technical assumptions, the valence band of both $\mathrm{C}$ and SRO with enhanced spatial resolution compared to that possible with photonics (Egerton, 2011). The relationship between EELS and MIT is summarized as follows.

1) Low-loss: In this regime, an EEL spectrum reflects the dielectric response (not an exact response, because
A Basic structure of TEM

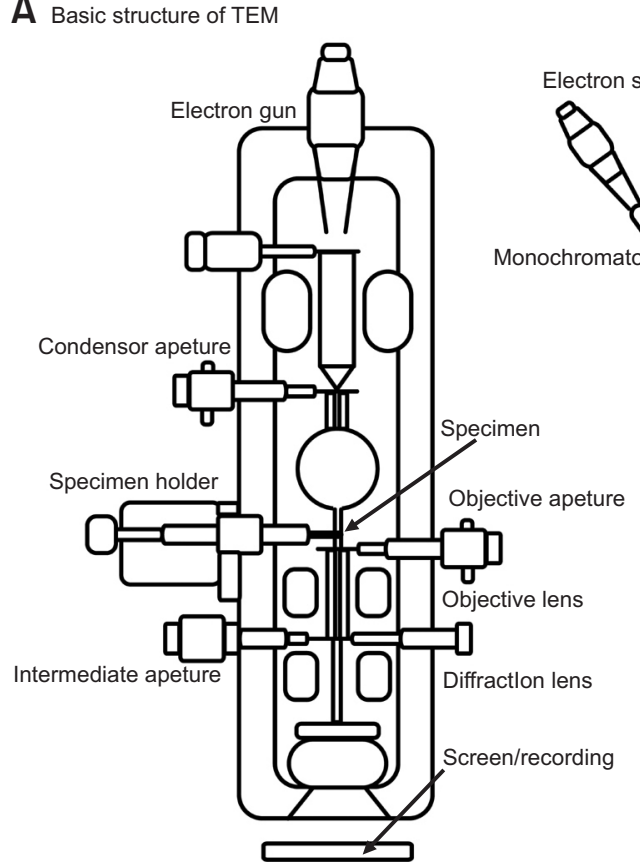

B EELS
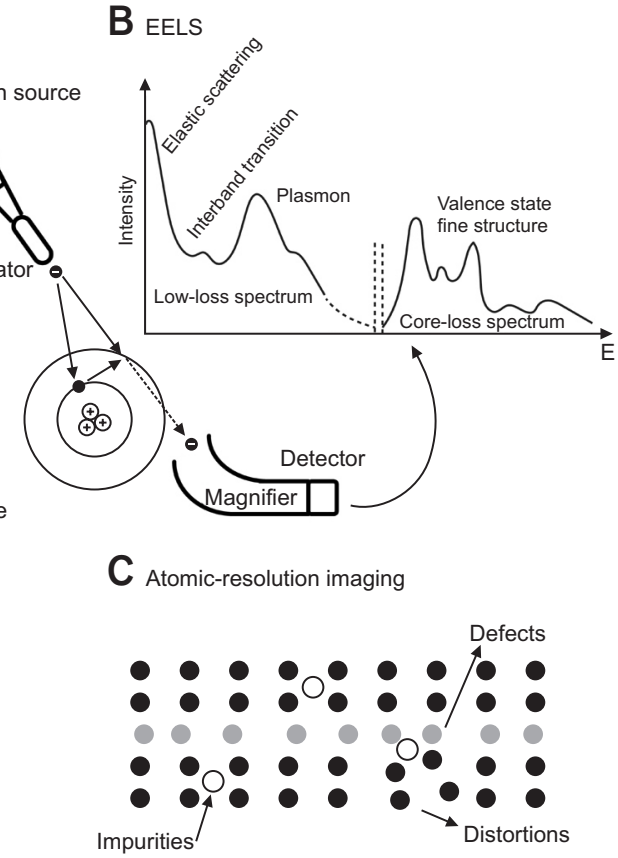

$D_{\text {EDS }}$

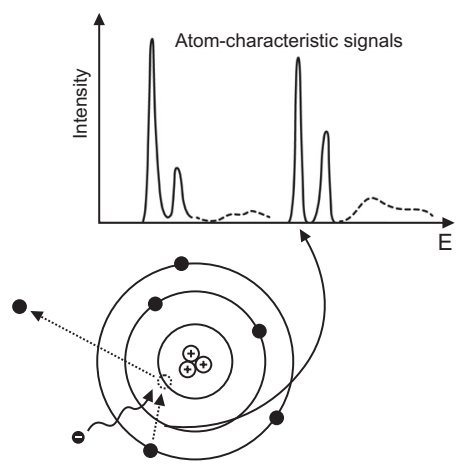

E

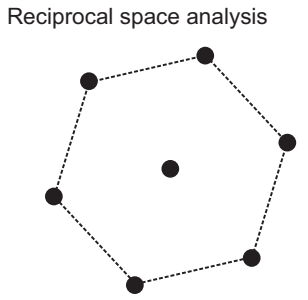

Fig. 7. Basic structure (A) and useful functions (B-E) of transmission electron microscopy (TEM). EELS, electron energy loss spectroscopy; EDS, electron dispersive spectroscopy. 


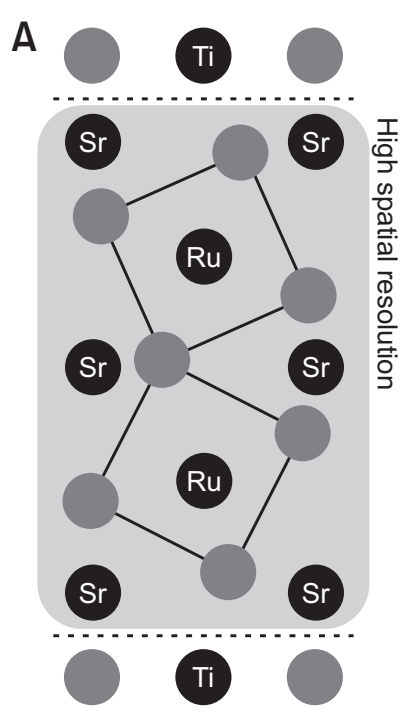

of the different properties of electrons and photons). Though the spectrum may suffer from unwanted plasmon excitations (e.g., Hohenester et al., 2009), reminiscences of $U$ and $\Delta$ will appear. The spectra themselves are directly connected to many-body physics (spectral function). The near-to-zero frequency region represents the correlations near the Fermi edge. If an elastic collision signal is deconvoluted fairly and the effect of low dimensionality is conspicuous, then the van Hove singularity of specific dimension would arise near the band gap in dielectric functions (Tompkins \& Irene, 2005).

2) Core-loss: The existence of charge transfer related with $\mathrm{Ru}$ is straightly demonstrated by $\mathrm{Ru} \mathrm{M} / \mathrm{L}$ edge spectra. Because of the strong Ru-O hybridization, the $\mathrm{O}$ K-edge spectrum will reflect the conduction band structure directly. For instance, we can observe the evolution of $\Delta_{\text {eff }}$ from the interface to the bulky region if it really opens the gap.

Comparing the band structures at different areas of the ultrathin system, one can entrench their own opinion about the role of interfaces and C. In this way, one can also judge that MIT either arises from the increasing portion of insulating $\mathrm{C}$ or remains in the intrinsic nature of SRO. In fact, we should examine the band structures with different lattice orientations to confirm the presence of dimensionality effects (i.e., the completely distinct boundary conditions for the electronic structure of SRO). TEM is clearly helpful in determining the order of each physical variable (e.g., lattice misfit) of SRO irrespective of the environment in $\mathrm{C} / \mathrm{SRO} / \mathrm{C}$ studied because the entire experimental procedures are integrated by TEM at once. For example, various characteristics of the physics of SRO that can be specified under the TEM environment are shown in Fig. 7. Because of the intriguing nature of the ultrathin SRO, we cannot prejudge or be certain about the existence of certain correlations. However, by examining components individually, we could determine the overall scale of each feature and, thus, the main origin of the MIT.

The interactions involving spins are ignored unless one precisely cares about the angular momentum multiplets. However, because ultrathin SRO retains its insulating behavior at high temperatures, where it has negligible net spin polarization, TEM and EELS will still show the localized bands. Accordingly, TEM-based studies are promising for studying the MIT in SRO.

\section{CONCLUSIONS}

Although DFT has been used to successfully describe the electronic properties of numerous materials, the origin of the behaviors of SRO has not fully elucidated because of this material's electronic correlations. In the simple Hubbard model, the effects of $U$ and $J$ are ascribed to such peculiar phenomena and the inclusion of two parameters are partially fruitful (recent DFT $+U$ and DMFT studies).

From the experimental side, the electrical anomalies are clearly observed through the spectra near the Fermi level, zero frequency optical responses, and unexpected suppression of ferromagnetism. Furthermore, the electronic state of $\mathrm{Ru}$ is well-known to strongly depend on the structure of the oxygen octahedron.

However, the MIT, at the zenith of the effects of electronic correlations, requires further study. It accompanies the complete shrinkage of partially filled bands and ferromagnetism in SRO. Both theoretically and experimentally, the issue of how the ensemble of strong perturbations (i.e., $U$, $J$, and $\Delta$ ) and the dimensionality can evoke MIT is not fully understood. 
TEM-based research is a promising solution, because it enables atomic-size analysis and electronic response of materials. Because of its high spatial resolution, TEM is sensitive to $\Delta$ and to the dimensionality (structural effects). With EELS, the spectral function of the $t_{2 g}$ band or other states can be observed. As such, compared with photonic spectroscopy, diffractometry, and some other microscopic techniques, TEM is suitable for demonstrating the very location of the origin of
MIT and, accordingly, the origin of the electronic correlations in SRO.

\section{CONFLICT OF INTEREST}

No potential conflict of interest relevant to this article was reported.

\section{REFERENCES}

Ahn J S, Bak J, Choi H S, Noh T W, Han J E, Bang Y, Cho J H, and Jia Q X (1999) Spectral Evolution in (Ca, $\mathrm{Sr}) \mathrm{RuO}_{3}$ near the Mott-Hubbard Transition. Phys. Rev. Lett. 82, 5321-5324.

Autieri C (2016) Antiferromagnetic and xy ferro-orbital order in insulating $\mathrm{SrRuO}_{3}$ thin films with SrO termination. J. Phys.: Condens. Matter 28, 42.

Bern F, Ziese M, Setzer A, Pippel E, Hesse D, and Vrejoiu I (2013) Structural, magnetic and electrical properties of $\mathrm{SrRuO}_{3}$ films and $\mathrm{SrRuO}_{3} / \mathrm{SrTiO}_{3}$ superlattices. J. Phys.: Condens. Matter 25, 49.

Chang S H, Chang Y J, Jang S Y, Jeong D W, Jung C U, Kim Y J, Chung J S, and Noh T W (2011) Thickness-dependent structural phase transition of strained $\mathrm{SrRuO}_{3}$ ultrathin films: the role of octahedral tilt. Phys. Rev. B 84, 10.

Chang Y J, Kim C H, Phark S H, Kim Y S, Yu J, and Noh T W (2009) Fundamental thickness limit of itinerant ferromagnetic $\mathrm{SrRuO}_{3}$ thin films. Phys. Rev. Lett. 103, 5.

Chen H, Millis A J, and Marianetti C A (2013) Engineering correlation effects via artificially designed oxide superlattices. Phys. Rev. Lett. 111, 11.

Choi K J, Baek S H, Jang H W, Belenky L J, Lyubchenko M, and Eom C $B$ (2010) Phase-transition temperatures of strained single-crystal $\mathrm{SrRuO}_{3}$ thin films. Adv. Mater. 22, 759-762.

Cox P A, Egdell R G, Goodenough J B, Hamnett A, and Naish C C (1983) The metal-to-semiconductor transition in ternary ruthenium (IV) oxides: a study by electron spectroscopy. J. Phys. C: Solid State Phys. 16, 6221-6239.

Dang H T, Mravlje J, Georges A, and Millis A J (2015) Electronic correlations, magnetism, and Hund's rule coupling in the ruthenium perovskites $\mathrm{SrRuO}_{3}$ and $\mathrm{CaRuO}_{3}$. Phys. Rev. B 91, 19.

de Boer J H and Verwey E J (1937) Semi-conductors with partially and with completely filled 3d-lattice bands. Proc. Phys. Soc. 49, 59-71.

de'Medici L, Hassan S R, Capone M, and Dai X (2009) Orbital-selective Mott transition out of band degeneracy lifting. Phys. Rev. Lett. 102, 12.

Dodge J S, Weber C P, Corson J, Orenstein J, Schlesinger Z, Reiner J W, and Beasley M R (2000) Low-frequency crossover of the fractional power-law conductivity in $\mathrm{SrRuO}_{3}$. Phys. Rev. Lett. 85, 4932-4935.

Egerton R F (2011) Electron Energy-Loss Spectroscopy in the Electron Microscope (Springer, New York).

Etz C, Maznichenko I V, Böttcher D, Henk J, Yaresko A N, Hergert W, Mazin I I, Mertig I, and Ernst A (2012) Indications of weak electronic correlations in $\mathrm{SrRuO}_{3}$ from first-principles calculations. Phys. Rev. $B$ 86, 6 .
Ferrero M, Becca F, Fabrizio M, and Capone M (2005) Dynamical behavior across the Mott transition of two bands with different bandwidths. Phys. Rev. B 72, 20.

Fujioka K, Okamoto J, Mizokawa T, Fujimori A, Hase I, Abbate M, Lin H J, Chen C T, Takeda Y, and Takano M (1997) Electronic structure of $\mathrm{SrRuO}_{3}$. Phys. Rev. B 56, 6380-6383.

Georges A, de'Medici L, and Mravlje J (2013) Strong correlations from Hund's coupling. Annu. Rev. Condens. Matter Phys. 4, 137-178.

Gorelov E, Karolak M, Wehling T O, Lechermann F, Lichtenstein A I, and Pavarini E (2010) Nature of the Mott transition in $\mathrm{Ca}_{2} \mathrm{RuO}_{4}$. Phys. Rev. Lett. 104, 22.

Grutter A, Wong F, Arenholz E, Liberati M, Vailionis A, and Suzuki Y (2010) Enhanced magnetism in epitaxial $\mathrm{SrRuO}_{3}$ thin films. Appl. Phys. Lett. 96, 8.

Gu M, Laverock J, Chen B, Smith K E, Wolf S A, and Lu J (2013) MetalInsulator transition induced in $\mathrm{CaVO}_{3}$ thin films. J. Appl. Phys. 113, 13.

Gu M, Wang K, Wang Y, Xie Q, Cai H, Zhang G P, and Wu X (2016) Enhancement of orbital ordering and spin polarization by controlling the dimensionality of the octahedra network. NPJ Quant. Mater. 1, 16011.

Gu M, Xie Q, Shen X, Xie R, Wang J, Tang G, Wu D, Zhang G P, and Wu $X$ S (2012) Magnetic ordering and structural phase transitions in a strained ultrathin $\mathrm{SrRuO}_{3} / \mathrm{SrTiO}_{3}$ superlattice. Phys. Rev. Lett. 109, 15.

Gunnarsson O, Koch E, and Martin R M (1996) Mott transition in degenerate Hubbard models: application to doped fullerenes. Phys. Rev. B 54, R11026-R11029.

Gupta K, Mandal B, and Mahadevan P (2014) Strain-induced MetalInsulator transition in ultrathin films of $\mathrm{SrRuO}_{3}$. Phys. Rev. B 90, 12.

Han Q, Dang H T, and Millis A J (2016) Ferromagnetism and correlation strength in cubic barium ruthenate in comparison to strontium and calcium ruthenate: a dynamical mean-field study. Phys. Rev. B 93, 15.

He J, Borisevich A, Kalinin S V, Pennycook S J, and Pantelides S T (2010) Control of octahedral tilts and magnetic properties of perovskite oxide heterostructures by substrate symmetry. Phys. Rev. Lett. 105, 22.

Hohenester U, Ditlbacher H, and Krenn J R (2009) Electron-energy-loss spectra of plasmonic nanoparticles. Phys. Rev. Lett. 103, 10.

Imada M, Fujimori A, and Tokura Y (1998) Metal-Insulator transitions. Rev. Mod. Phys. 70, 1039-1263. 
Ishigami K, Yoshimatsu K, Toyota D, Takizawa M, Yoshida T, Shibata G, Harano T, Takahashi Y, Kadono T, Verma V K, Singh V R, Takeda Y, Okane T, Saitoh Y, Yamagami H, Koide T, Oshima M, Kumigashira H, and Fujimori A (2015) Thickness-dependent magnetic properties and strain-induced orbital magnetic moment in $\mathrm{SrRuO}_{3}$ thin films. Phys. Rev. B 92, 6 .

Jang H W, Felker D A, Bark C W, Wang Y, Niranjan M K, Nelson C T, Zhang Y, Su D, Folkman C M, Baek S H, Lee S, Janicka K, Zhu Y, Pan X Q, Fong D D, Tsymbal E Y, Rzchowski M S, and Eom C B (2011) Metallic and insulating oxide interfaces controlled by electronic correlations. Science 331, 886-889.

Jeng H T, Lin S H, and Hsue C S (2006) Orbital ordering and Jahn-Teller distortion in perovskite ruthenate $\mathrm{SrRuO}_{3}$. Phys. Rev. Lett. 97, 6 .

Jeong D W, Choi H C, Kim C H, Chang S H, Sohn C H, Park H J, Kang T D, Cho D Y, Baek S H, Eom C B, Shim J H, Yu J, Kim K W, Moon S J, and Noh T W (2013) Temperature evolution of itinerant ferromagnetism in $\mathrm{SrRuO}_{3}$ probed by optical spectroscopy. Phys. Rev. Lett. 110, 24.

Kacedon D B, Rao R A, and Eom C B (1997) Magnetoresistance of epitaxial thin films of ferromagnetic metallic oxide $\mathrm{SrRuO}_{3}$ with different domain structures. Appl. Phys. Lett. 71, 1724-1726.

Kanbayasi A (1976) Magnetic properties of $\mathrm{SrRuO}_{3}$ single crystal. J. Phys. Soc. Jpn. 41, 1876-1878.

Kennedy B J and Hunter B A (1998) High-temperature phases of $\mathrm{SrRuO}_{3}$. Phys. Rev. B 58, 653-658.

Kostic P, Okada Y, Collins N C, Schlesinger Z, Reiner J W, Klein L, Kapitulnik A, Geballe T H, and Beasley M R (1998) Non-Fermi-liquid behavior of $\mathrm{SrRuO}_{3}$ : evidence from infrared conductivity. Phys. Rev. Lett. 81, 2498-2501.

Kim B and Min B I (2014) Termination-dependent electronic and magnetic properties of ultrathin $\mathrm{SrRuO}_{3}(111)$ films on $\mathrm{SrTiO}_{3}$. Phys. Rev. B 89, 19.

Kim H D, Noh H J, Kim K H, and Oh S J (2004) Core-level X-ray photoemission satellites in ruthenates: A new mechanism revealing the Mott transition. Phys. Rev. Lett. 93, 12.

Kim J, Chung J, and Oh S J (2005a) In situ photoemission study on $\mathrm{SrRuO}_{3}$ $/ \mathrm{SrTiO}_{3}$ films grown by pulsed laser deposition. Phys. Rev. B 71, 12.

Kim K W, Lee J S, Noh T W, Lee S R, and Char K (2005b) Metal-Insulator transition in a disordered and correlated $\mathrm{SrTi}_{1-\mathrm{x}} \mathrm{Ru}_{\mathrm{x}} \mathrm{O}_{3}$ system: changes in transport properties, optical spectra, and electronic structure. Phys. Rev. B 71, 12.

Kim M and Min B I (2015) Nature of itinerant ferromagnetism of $\mathrm{SrRuO}_{3}$ : A DFT+DMFT study. Phys. Rev. B 91, 20.

Kimber S A J, Rodgers J A, Wu H, Murray C A, Argyriou D N, Fitch A N, Khomskii D I, and Attfield J P (2009) Metal-Insulator transition and orbital order in $\mathrm{PbRuO}_{3}$. Phys. Rev. Lett. 102, 4.

Koga A, Kawakami N, Rice T M, and Sigrist M (2004) Orbital-selective Mott transitions in the degenerate Hubbard model. Phys. Rev. Lett. 92, 21.

Koster G, Klein L, Siemons W, Rijnders G, Dodge J S, Eom C B, Blank D H A, and Beasley M R (2012) Structure, physical properties, and applications of $\mathrm{SrRuO}_{3}$ thin films. Rev. Mod. Phys. 84, 253-298.

Kotliar G and Vollhardt D (2004) Strongly correlated materials: insights from dynamical mean-field theory. Phys. Today 57, 53-60.

Kumigashira H, Minohara M, Takizawa M, Fujimori A, Toyota D, Ohkubo I, Oshima M, Lippmaa M, and Kawasaki M (2008) Interfacial electronic structure of $\mathrm{SrTiO}_{3} / \mathrm{SrRuO}_{3}$ heterojuctions studied by in situ photoemission spectroscopy. Appl. Phys. Lett. 92, 12.
Lee J S, Lee Y S, Noh T W, Char K, Park J, Oh S J, Park J H, Eom C B, Takeda $\mathrm{T}$, and Kanno $\mathrm{R}$ (2001) Optical investigation of the electronic structures of $\mathrm{Y}_{2} \mathrm{Ru}_{2} \mathrm{O}_{7}, \mathrm{CaRuO}_{3}, \mathrm{SrRuO}_{3}$, and $\mathrm{Bi}_{2} \mathrm{Ru}_{2} \mathrm{O}_{7}$. Phys. Rev. $B$ 64, 24.

Liebsch A (2003a) Quasi-particle spectra of perovskites: enhanced Coulomb correlations at surfaces. Eur. Phys. J. B 32, 477-487.

Liebsch A (2003b) Surface versus bulk Coulomb correlations in photoemission spectra of $\mathrm{SrVO}_{3}$ and $\mathrm{CaVO}_{3}$. Phys. Rev. Lett. 90, 9.

Liebsch A, Ishida H, and Bihlmayer G (2005) Coulomb correlations and orbital polarization in the Metal-Insulator transition of $\mathrm{VO}_{2}$. Phys. Rev. B 71, 8 .

Lin P A, Jeng H T, and Hsue C S (2008) Electronic structure and orbital ordering of $\mathrm{SrRu}_{1-\mathrm{x}} \mathrm{Ti}_{x} \mathrm{O}_{3}$ : GGA+U investigations. Phys. Rev. B 77, 8 .

Liu Z Q, Ming Y, Lü W M, Huang Z, Wang $X$, Zhang $B$ M, Feng $Y$ P, Venkatesan T, and Ariando (2012) Tailoring the electronic properties of $\mathrm{SrRuO}_{3}$ films in $\mathrm{SrRuO}_{3} / \mathrm{LaAlO}_{3}$ superlattices. Appl. Phys. Lett. 101, 22.

Lu W, Song W D, He K, Chai J, Sun C J, Chow G M, and Chen J S (2013a) The role of octahedral tilting in the structural phase transition and magnetic anisotropy in $\mathrm{SrRuO}_{3}$ thin film. J. Appl. Phys. 113, 6.

Lu W, Yang P, Song W D, Chow G M, and Chen J S (2013b) Control of oxygen octahedral rotations and physical properties in $\mathrm{SrRuO}_{3}$ films. Phys. Rev. B 88, 21.

Mahadevan P, Aryasetiawan F, Janotti A, and Sasaki T (2009) Evolution of the electronic structure of a ferromagnetic metal: case of $\mathrm{SrRuO}_{3}$. Phys. Rev. B 80, 3.

Maiti $\mathrm{K}$ and Singh R S (2005) Evidence against strong correlation in $4 d$ transition-metal oxides $\mathrm{CaRuO}_{3}$ and $\mathrm{SrRuO}_{3}$. Phys. Rev. B 71, 16.

Maiti K, Singh R S, and Medicherla V R R (2007a) Evolution of a band insulating phase from a correlated metallic phase. Phys. Rev. B 76, 16.

Maiti K, Singh R S, and Medicherla V R R (2007b) Observation of particle hole asymmetry and phonon excitations in non-Fermi-liquid systems: a high-resolution photoemission study of ruthenates. Europhys. Lett. 78, 1.

Mazin I I and Singh D J (1997) Electronic structure and magnetism in Rubased perovskites. Phys. Rev. B 56, 2556-2571.

Mott N (1990) On Metal-Insulator transitions. J. Solid State Chem. 88, 5-7.

Okamoto J, Mizokawa T, Fujimori A, Hase I, Nohara M, Takagi H, Takeda Y, and Takano M (1999) Correlation effects in the electronic structure of $\mathrm{SrRuO}_{3}$. Phys. Rev. B 60, 2281-2285.

Park J, Oh S J, Park J H, Kim D M, and Eom C B (2004) Electronic structure of epitaxial $(\mathrm{Sr}, \mathrm{Ca}) \mathrm{RuO}_{3}$ films studied by photoemission and X-ray absorption spectroscopy. Phys. Rev. B 69, 8.

Poteryaev A I, Ferrero M, Georges A, and Parcollet O (2008) Effect of crystal-field splitting and interband hybridization on the MetalInsulator transitions of strongly correlated systems. Phys. Rev. B $\mathbf{7 8}$, 4.

Poteryaev A I, Tomczak J M, Biermann S, Georges A, Lichtenstein A I, Rubtsov A N, Saha-Dasgupta T, and Andersen O K (2007) Enhanced crystal-field splitting and orbital-selective coherence induced by strong correlations in $\mathrm{V}_{2} \mathrm{O}_{3}$. Phys. Rev. B 76, 8 .

Rondinelli J M, Caffrey N M, Sanvito S, and Spaldin N A (2008) Electronic properties of bulk and thin film $\mathrm{SrRuO}_{3}$ : search for the MetalInsulator transition. Phys. Rev. B 78, 15.

Rozenberg M J, Kotliar G, and Kajueter H (1996) Transfer of spectral 
weight in spectroscopies of correlated electron systems. Phys. Rev. $B$ 54, 8452-8468.

Ryee S, Jang S W, Kino H, Kotani T, and Han M J (2016) Quasiparticle self-consistent $\mathrm{GW}$ calculation of $\mathrm{Sr}_{2} \mathrm{RuO}_{4}$ and $\mathrm{SrRuO}_{3}$. Phys. Rev. $B$ 93, 7.

Shai D E, Adamo C, Shen D W, Brooks C M, Harter J W, Monkman E J, Burganov B, Schlom D G, and Shen K M (2013) Quasiparticle mass enhancement and temperature dependence of the electronic structure of ferromagnetic $\mathrm{SrRuO}_{3}$ thin films. Phys. Rev. Lett. 110, 8.

Shen X, Qiu X, Su D, Zhou S, Li A, and Wu D (2015) Thickness-dependent Metal-Insulator transition in epitaxial $\mathrm{SrRuO}_{3}$ ultrathin films. J. Appl. Phys. 117, 1.

Si L, Zhong Z, Tomczak J M, and Held K (2015) Route to roomtemperature ferromagnetic ultrathin $\mathrm{SrRuO}_{3}$ films. Phys. Rev. B 92, 4.

Siemons W, Koster G, Vailionis A, Yamamoto H, Blank D H, and Beasley M R (2007) Dependence of the electronic structure of $\mathrm{SrRuO}_{3}$ and its degree of correlation on cation off-stoichiometry. Phys. Rev. B 76, 7.

Takizawa M, Toyota D, Wadati H, Chikamatsu A, Kumigashira H, Fujimori A, Oshima M, Fang Z, Lippmaa M, Kawasaki M, and Koinuma H (2005) Manifestation of correlation effects in the photoemission spectra of $\mathrm{Ca}_{1-\mathrm{x}} \mathrm{Srx} \mathrm{RuO}_{3}$. Phys. Rev. B 72, 6.

Tian W, Haeni J H, Schlom D G, Hutchinson E, Sheu B L, Rosario M M, Schiffer P, Liu Y, Zurbuchen M A, and Pan X Q (2007) Epitaxial growth and magnetic properties of the first five members of the layered $\mathrm{Sr}_{\mathrm{n}+1}$ $\mathrm{Ru}_{n} \mathrm{O}_{3 n+1}$ oxide series. Appl. Phys. Lett. 90, 2.

Tompkins $\mathrm{H}$, and Irene E A (2005) Handbook of ellipsometry (William Andrew).

Toyota D, Ohkubo I, Kumigashira H, Oshima M, Ohnishi T, Lippmaa M, Kawasaki M, and Koinuma $\mathrm{H}$ (2006) Ferromagnetism stabilization of ultrathin $\mathrm{SrRuO}_{3}$ films: thickness-dependent physical properties. J. Appl. Phys. 99, 8.

Toyota D, Ohkubo I, Kumigashira H, Oshima M, Ohnishi T, Lippmaa M, Takizawa M, Fujimori A, Ono K, Kawasaki M, and Koinuma H (2005) Thickness-dependent electronic structure of ultrathin $\mathrm{SrRuO}_{3}$ films studied by in situ photoemission spectroscopy. Appl. Phys. Lett. 87, 16.

Vailionis A, Siemons W, and Koster G (2008) Room temperature epitaxial stabilization of a tetragonal phase in $\mathrm{ARuO}_{3}(\mathrm{~A}=\mathrm{Ca}$ and $\mathrm{Sr})$ thin films. Appl. Phys. Lett. 93, 5.
Verissimo-Alves M, García-Fernández P, Bilc D I, Ghosez P, and Junquera $\mathrm{J}$ (2012) Highly confined spin-polarized two-dimensional electron gas in $\mathrm{SrTiO}_{3} / \mathrm{SrRuO}_{3}$ superlattices. Phys. Rev. Lett. 108, 10.

Wang G T, Zhang M P, Yang Z X, and Fang Z (2009) Orbital orderings and optical conductivity of $\mathrm{SrRuO}_{3}$ and $\mathrm{CaRuO}_{3}$ : first-principles studies. J. Phys.: Condens. Matter 21, 26.

Williams A J, Gillies A, Attfield J P, Heymann G, Huppertz H, Martinez-Lope $\mathrm{M} \mathrm{J}$, and Alonso J A (2006) Charge transfer and antiferromagnetic insulator phase in $\mathrm{SrRu}_{1-\mathrm{x}} \mathrm{Cr}_{\mathrm{x}} \mathrm{O}_{3}$ perovskites: solid solutions between two itinerant electron oxides. Phys. Rev. B 73, 10.

Woodward P M (1997) Octahedral tilting in perovskites. II. structure stabilizing forces. Acta Crystallogr. Sect. B: Struct. Sci. 53, 44-66.

Xia J, Siemons W, Koster G, Beasley M R, and Kapitulnik A (2009) Critical thickness for itinerant ferromagnetism in ultrathin films of $\mathrm{SrRuO}_{3}$. Phys. Rev. B 79, 14.

Yang H F, Fan C C, Liu Z T, Yao Q, Li M Y, Liu J S, Jiang M H, and Shen D W (2016) Comparative angle-resolved photoemission spectroscopy study of $\mathrm{CaRuO}_{3}$ and $\mathrm{SrRuO}_{3}$ thin films: pronounced spectral weight transfer and possible precursor of lower Hubbard band. Phys. Rev. $B$ 94, 11.

Yang H F, Liu Z T, Fan C C, Yao Q, Xiang P, Zhang K L, Li M Y, Li H, Liu J S, Shen D W, and Jiang M H (2016) Origin of the kink in the band dispersion of the ferromagnetic perovskite $\mathrm{SrRuO}_{3}$ : electron-phonon coupling. Phys. Rev. B 93, 12.

Yoo H K, Chang Y J, Moreschini L, Kim H D, Sohn C H, Sinn S, Oh J S, Kuo C T, Bostwick A, Rotenberg E, and Noh T W (2015) Insulating-layer formation of metallic $\mathrm{LaNiO}_{3}$ on Nb-doped $\mathrm{SrTiO}_{3}$ substrate. Appl. Phys. Lett. 106, 12.

Yoshimatsu K, Okabe T, Kumigashira H, Okamoto S, Aizaki S, Fujimori A, and Oshima M (2010) Dimensional-crossover-driven Metal-Insulator transition in $\mathrm{SrVO}_{3}$ ultrathin films. Phys. Rev. Lett. 104, 14.

Yoshimatsu K, Horiba K, Kumigashira H, Yoshida T, Fujimori A, and Oshima M (2011). Metallic quantum well states in artificial structures of strongly correlated oxide. Science $\mathbf{3 3 3}, \mathbf{3 1 9}-322$.

Zhong Z, Wallerberger M, Tomczak J M, Taranto C, Parragh N, Toschi A, Sangiovanni G, and Held K (2015) Electronics with correlated oxides: $\mathrm{SrVO}_{3} / \mathrm{SrTiO}_{3}$ as a Mott transistor. Phys. Rev. Lett. 114, 24.

Zhong Z, Zhang Q, and Held K (2013) Quantum confinement in perovskite oxide heterostructures: tight binding instead of a nearly free electron picture. Phys. Rev. B 88, 12. 